\title{
Fourier-Stokes polarimetry of fields corresponding to linearly and circularly polarized birefringent protein networks
}

\author{
A.O. Karachevtsev \\ Chernivtsi National University named after Yuri Fed'kovych, \\ Department for Optics and Spectroscopy, \\ 2 Kotsyubyns 'kogo str., 58001 Chernivtsi, Ukraine. \\ Phone: +38067 7830572
}

\begin{abstract}
Considered in this work are the possibilities to use the method of FourierStokes polarimetry for diagnostics of optical anisotropy inherent to polycrystalline networks of biological layers. The authors have offered a generalized model for optical anisotropy of histological sections taken from biological tissues and films of biological liquids. Polarization maps for Fourier spectra of laser images describing the phaseinhomogeneous objects have been investigated. Grounded was application of the method for consistent spatial-frequency filtration of polarization maps formed by linear and circular birefringent of biological crystals.
\end{abstract}

Keywords: polarization, Jones matrix, optical anisotropy, biological layer, polarization map, Fourier spectrum, spatial-frequency filtration.

Manuscript received 08.02.12; revised version received 07.06.12; accepted for publication 14.06.12; published online 25.09.12.

\section{Introduction}

Among the variety of systems for diagnostics of optical anisotropy of biological objects [1-3], a new direction laser polarimetry of biological tissue images has been separated [4, 5]. In this approach, the analysis of the obtained data is based on approximation of linear birefringence observed in polycrystalline protein networks. Using the above model approach, the authors found interrelation between the set of statistical moments of the first to fourth orders that characterize distributions of azimuths and ellipticity of polarizations in laser images as well as parameters of anisotropy inherent to the layers of biological tissues [6-7]. As a result, developed was the method for polarization mapping and successfully realized was the diagnostics of oncological (malignant) changes in human biological tissue $[8,9]$. To further develop the methods of laser polarimetric diagnostics, it seems topical to differentiate various mechanisms of optical anisotropy. Up to date, the above method of polarization mapping is not able to solve this problem. One of the possible solutions lies in using the consistent spatial-frequency filtration of laser images [10]. The idea of this approach, which is novelty in biomedical applications, is based on filtration of spatial-frequency spectra for polarization images of networks including biological crystals with different mechanisms of anisotropy. Optical realization of this method consists of the following stages: direct Fourier transform, spatial-frequency and polarization-phase filtration, inverse Fourier transform of biological layer laser images.

This work is aimed at development of a generalized model of phase anisotropy to describe formation of fields inherent to scattered laser radiation and, with account of this model, substantiation of new methods for Fourier-Stokes polarimetry as well as consistent polarization-phase filtration of manifestations inherent to linear and circular birefringence for diagnostics of the structure of polycrystalline networks observed in biological tissues and liquids.

\section{Model conceptions and computer modeling the processes of formation of polarization- inhomogeneous images and their Fourier spectra}

As a basis for our investigations, we took the following model conceptions: 
1. Substance of optically anisotropic component of biological tissue possesses some generalized anisotropy that is a superposition of linear and circular birefringence.

2. From the analytical viewpoint, processes of interaction of laser radiation with this substance are described using the Jones-matrix operators that are the most adequate and adapted to the processes of amplitude-phase modulation of coherent radiation.

3. Polarization-inhomogeneous distributions of laser radiation fields in the far Fourier diffraction zone can be determined using the Kirhhoff integrals.

The process of transformation of laser radiation parameters by an optically anisotropic layer is described with the following matrix equation

$$
\{J\}=\{D\}\{C\} \text {. }
$$

Here, $\{D\}$ is the Jones matrix for linear birefringence, $\{C\}$ - Jones matrix for optical activity

$\{D\}=\left[\begin{array}{ll}d_{11} & d_{12} \\ d_{21} & d_{22}\end{array}\right]$,

where

$$
\begin{aligned}
& \left\{\begin{array}{c}
d_{11}=\sin ^{2} \kappa+\cos ^{2} \kappa \exp (-i \varphi) ; \\
d_{22}=\cos ^{2} \kappa+\sin ^{2} \kappa \exp (-i \varphi) ; \\
d_{12}=d_{21}=\sin \kappa \cos \kappa(1-\exp (-i \varphi)) .
\end{array}\right. \\
& \{C\}=\left[\begin{array}{ll}
c_{11} & c_{12} \\
c_{21} & c_{22}
\end{array}\right],
\end{aligned}
$$

with

$$
\left\{\begin{array}{c}
c_{11}=\cos \psi ; \\
c_{22}=\cos \psi ; \\
c_{12}=-c_{21}=\sin \psi .
\end{array}\right.
$$

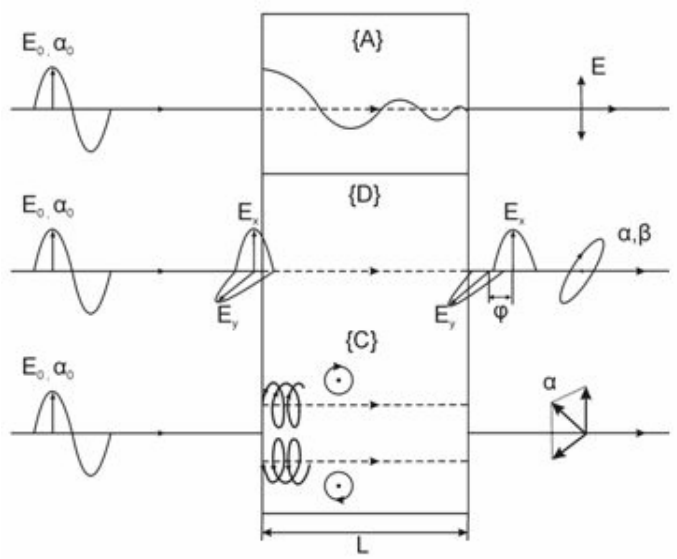

Fig. 1. On analysis of model conceptions. $E_{0}, \alpha_{0}$ are the amplitude and azimuth of probing beam polarization; $E_{x}, E_{y}, \alpha$, $\beta, \varphi$ - orthogonal components, azimuth, ellipticity and phase of the object beam $E ;\{A\},\{D\},\{C\}$ - Jones matrixes for isotropic, linear and circular birefringent components of a biological layer.
In a detailed look, the equation (1) can be rewritten as

$$
\begin{aligned}
& \left(\begin{array}{c}
E_{x} e^{i \varphi_{x}} \\
E_{y} e^{i \varphi_{y}}
\end{array}\right)=\left[\begin{array}{cc}
\cos ^{2} \kappa+e^{-i \varphi} \sin ^{2} \kappa & \cos \kappa \sin \kappa\left(1-e^{-i \varphi}\right) \\
\cos \kappa \sin \kappa\left(1-e^{-i \varphi}\right) & \sin ^{2} \kappa+e^{-i \varphi} \cos ^{2} \kappa
\end{array}\right] \times \\
& \times\left[\begin{array}{cc}
\sin ^{2} \psi & \sin \psi \cos \psi \\
\sin \psi \cos \psi & \cos ^{2} \psi
\end{array}\right]\left(\begin{array}{l}
E_{0 x} e^{i \varphi_{0 x}} \\
E_{0 x} e^{i \varphi_{0 y}}
\end{array}\right) .
\end{aligned}
$$

From (6), one can obtain expressions for orthogonal complex components of the laser wave amplitude in an arbitrary point belonging to the studied crystalline network:

$$
\begin{aligned}
& E_{x}=E_{0 x}\left[\left(\cos ^{2} \kappa+e^{-i \gamma} \sin ^{2} \kappa\right) \cos \psi+\right. \\
& \left.+\cos \kappa \sin \kappa\left(1-e^{-i \gamma}\right) \sin \psi\right]+ \\
& +e^{i \varphi_{0 y}} E_{0 y}\left[\left(\cos ^{2} \kappa+e^{-i \gamma} \sin ^{2} \kappa\right) \sin \psi+\right. \\
& \left.\cos \kappa \sin \kappa\left(1-e^{-i \gamma}\right) \cos \psi\right] \\
& E_{y}=E_{0 x}\left[\cos \kappa \sin \kappa\left(1-e^{-i \gamma}\right) \sin \psi+\right. \\
& \left.+\left(\cos ^{2} \kappa+e^{-i \gamma} \sin ^{2} \kappa\right)\left(1-e^{-i \gamma}\right) \cos \psi\right]+ \\
& +e^{i \varphi_{0 y}} E_{0 y}\left[\cos \kappa \sin \kappa\left(1-e^{-i \gamma}\right) \sin \psi+\right. \\
& \left.+\left(\cos ^{2} \kappa+e^{-i \gamma} \sin ^{2} \kappa\right) \cos \psi\right]
\end{aligned}
$$

In the case of placing the biological tissue layer into the objective focal plane, one can obtain the following distribution of complex amplitudes in the Fourier plane:

$U\left(m^{*}, n^{*}\right)=\frac{A}{i \lambda f} \int_{-\infty}^{+\infty} E(m, n) \exp \left[-i \frac{2 \pi}{\lambda f}\left(m n^{*}+n n^{*}\right) d m d n\right]$

Here, $f$ is the objective focal distance; $\lambda-$ wavelength of laser radiation; $m, n$ and $m^{*}, n^{*}$ are coordinates of points in the image and Fourier planes, respectively.

\section{Computer modeling the processes of polarization map formation}

To determine the main interrelations between opticaland-geometrical characteristics of birefringent networks inherent to biological crystals and coordinate distributions for azimuths and ellipticities of polarization in the Fourier plane, we performed computer modeling.

As virtual birefringent networks, we used two types of objects, namely: i) a network of birefringent cylinders ordered in the direction of optical axes and placed in the same plane; ii) an ensemble of birefringent globes, centers of which are located in the same plane.

The results of computer modeling for distributions of polarization azimuths inherent to laser images of cylindrical and spherical birefringent networks in the Fourier plane are shown in Fig. 2. 


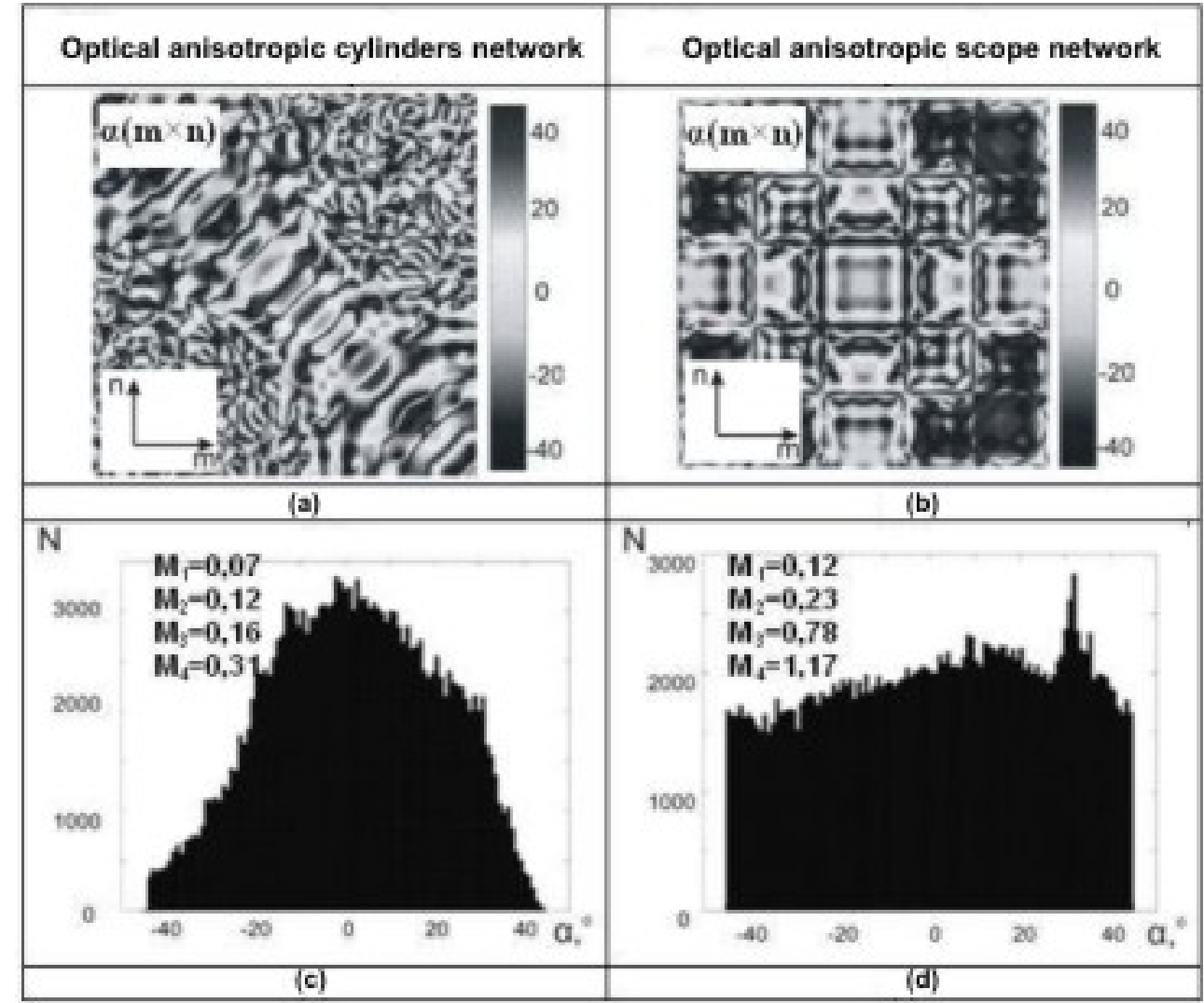

Fig. 2. Polarization maps for Fourier spectra and their statistical parameters.

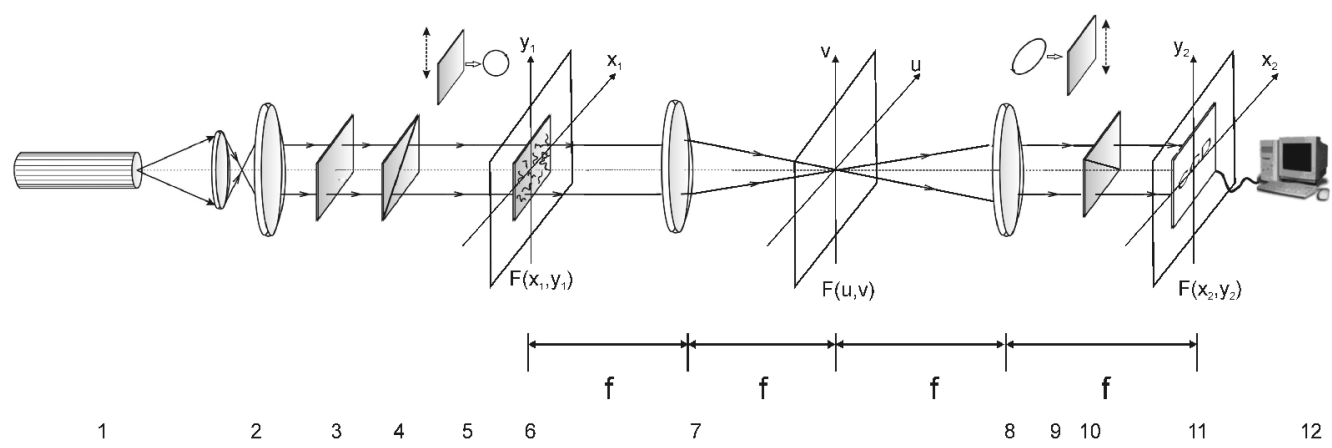

Fig. 3. Optical scheme of the polarization Fourier spectrometer where: 1 - He-Ne laser; 2 - collimator; 3 - stationary quarterwave plate; 4, 10 - polarizer and analyzer, respectively; 5, 9- mechanically movable quarter-wave plates; 6 - object under study; 7, 8 - micro-objectives; 11 - CCD camera; 12 - personal computer.

Performed by us complex statistical, correlation and fractal analyses for coordinate distributions of polarization states for scattered laser radiation in the Fourier plane revealed:

1. Polarization-inhomogeneous structure in Fourier patterns of laser images for ensembles of optically anisotropic birefringent crystals.

2. Coordinate inhomogeneity of Fourier patterns for laser images, which is caused by superposition of differently polarized partial laser waves.

3. Scale-self-similar multifractal structure for azimuth distributions and polarization ellipticities in the
Fourier plane for the field of scattered laser radiation.

4. Dependence of the set for statistical, correlation and fractal parameters on optical-and-geometrical parameters of virtual ordered cylinders and spheres.

\section{Experimental application of the Fourier method of polarization mapping}

Represented in Fig. 3 is the optical scheme of experimental setup for Fourier spectrometry of laser images inherent to biological tissues.

\section{(C) 2012, V. Lashkaryov Institute of Semiconductor Physics, National Academy of Sciences of Ukraine}




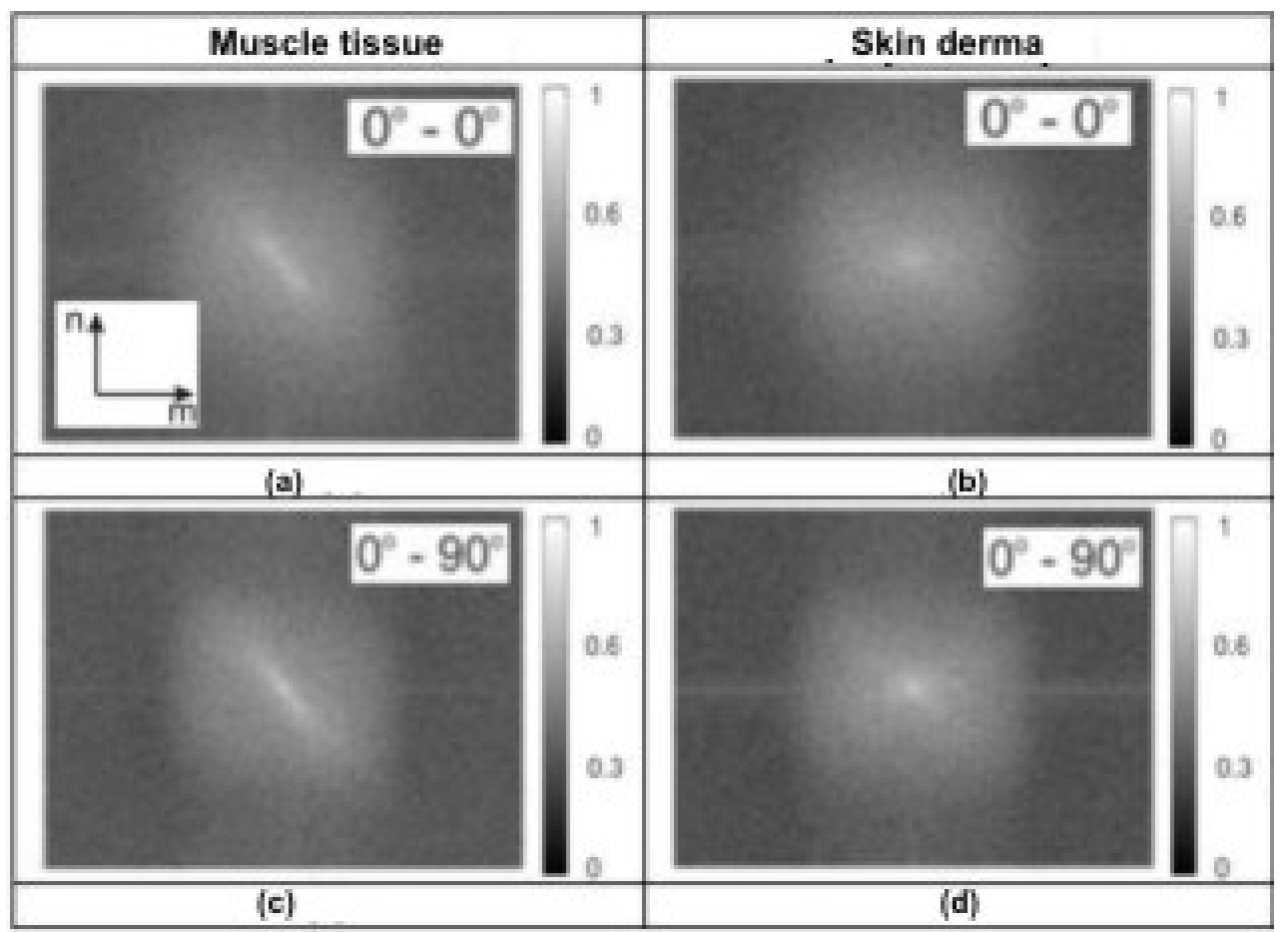

Fig. 4. Polarization filtered out Fourier spectra of laser images corresponding to histological sections of biological tissues.

Illumination was performed using a parallel $(\varnothing=$ $\left.10^{4} \mu \mathrm{m}\right) \mathrm{He}-\mathrm{Ne}$ laser beam $(\lambda=0.6328 \mu \mathrm{m}, \mathrm{W}=$ $5.0 \mathrm{~mW}$ ). The polarization illuminator consists of the quarter-wave plates 3 and 5 as well as polarizer 4 , which provides formation of the laser beam with an arbitrary azimuth $0^{\circ} \leq \alpha_{0} \leq 180^{\circ}$ or ellipticity of polarization $0^{\circ} \leq \beta_{0} \leq 90^{\circ}$.

Histological sections of biological tissue samples were placed in the focal plane of the micro-objective 7 (focal distance $f=450 \mu \mathrm{m}$, magnification $4 \mathrm{X}$ ), their images were projected into the plane of the lightsensitive area $(800 \times 600$ pixels $)$ of CCD-camera 10 $(P=m \times n=600$ pix $\times 800$ pix $)$ that was also placed at the focal distance and provided the range of measurements for structural elements in the Fourier spectrum of biological tissue images for the following geometric sizes: between 2 to $2000 \mu \mathrm{m}$.

Our experimental conditions were chosen in such a manner that enabled us to practically eliminate spatialangular filtration when forming the Fourier spectra of biological tissue laser images. It was reached by matching the angular characteristics for indicatrixes of light scattering in biological tissue samples $\left(\Omega_{5 T} \approx 16^{0}\right)$ with the angular aperture of the micro-objective $\left(\Delta \omega=20^{0}\right)$. Here, $\Omega_{5 T}$ is the angular cone of indicatrixes, where $98 \%$ of the total energy belonging to scattered radiation is concentrated.

The analysis of polarization structure in the Fourier spectra corresponding to images of histological sections of biological tissues was performed using the polarizer 10 and the quarter-wave 9 .

We investigated the samples of histological sections taken from biological tissues of two types:

- muscular tissue - as a system of ordered along definite direction birefringent myosin fibrils;

- skin derma - as a system of disordered birefringent collagen fibrils.

It has been ascertained that spatially-ordered fibrilar network is characterized by asymmetric shape of Fourier images (Figs $4 \mathrm{a}$ and $4 \mathrm{c}$ ) in polarization filtered out images. In the case of disordered birefringent network (Figs 4b and 4d), the Fourier patterns are azimuth-symmetrical.

From the quantitative viewpoint, this polarizationinhomogeneous structure in Fourier patterns of laser images obtained for histological sections is illustrated with histograms for distributions of polarization azimuths in Fig. 5.

The ordered network is characterized by availability of several extremes related with predominant directions of folding of myosin fibrils. The disordering process observed for collagen fibrils network in healthy skin derma is manifested as re-distribution of extremes in the respective histogram to the side of more equiprobable structure. The obtained data correlate well with those of computer modeling. Quantitatively, the differences between coordinate distributions for polarization azimuths are illustrated with values of statistical moments of the first to fourth orders that are adduced in Table 1.

\section{(C) 2012, V. Lashkaryov Institute of Semiconductor Physics, National Academy of Sciences of Ukraine}




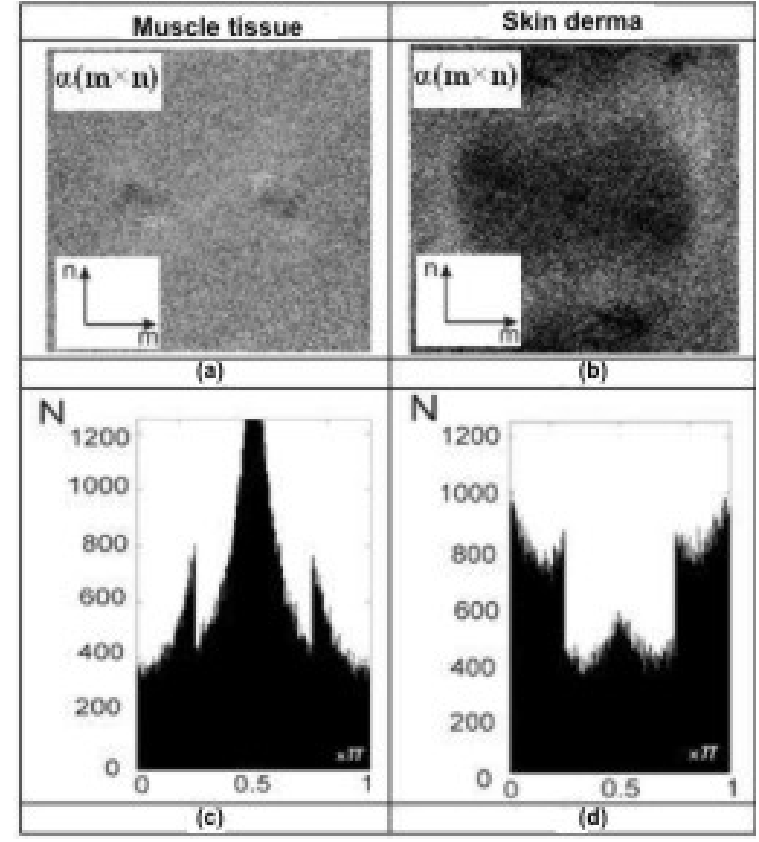

Fig. 5. Polarization maps $(a, b)$ and histograms of distributions (c, d) of polarization azimuths in Fourier spectra corresponding to images of muscular tissue (a, c) and skin derma (b, d).

Table 1. Statistical moments of the first to fourth orders for polarization azimuth distributions.

\begin{tabular}{|c|c|c|}
\hline $\mathrm{M}_{\mathrm{i}}$ & $\begin{array}{c}\text { Muscular tissue } \\
(\mathrm{q}=19)\end{array}$ & $\begin{array}{c}\text { Skin derma } \\
(\mathrm{q}=18)\end{array}$ \\
\hline $\mathrm{M}_{1}$ & $0.14 \pm 0.01$ & $0.17 \pm 0.01$ \\
\hline $\mathrm{M}_{2}$ & $0.11 \pm 0.02$ & $0.24 \pm 0.01$ \\
\hline $\mathrm{M}_{3}$ & $0.21 \pm 0.04$ & $0.46 \pm 0.01$ \\
\hline $\mathrm{M}_{4}$ & $0.43 \pm 0.05$ & $1.28 \pm 0.01$ \\
\hline
\end{tabular}

It has been ascertained that statistical moments of the third and fourth orders are the most sensitive to orientation changes in optical axes distributions inherent to biological crystals. The differences between them reach 2 and 3 times, respectively.

The next step was studying the possibility to detect changes in birefringence by using experimental investigation of the correlation structure inherent to Fourier patterns of maps for azimuths and ellipticities in laser images. As objects for experimental investigations, we chose two groups of experimental samples prepared in the form of optically thin histological sections by using a freezing microtome in accord with the standard technique. The first group of the samples $(N=17)$ was histological sections of biopsy taken from benign tumor of rectum. The second group of the samples $(N=16)$ was histological sections of biopsy taken from malignant tumor of rectum. The topicality of development of the methods in Fourier-Stokes polarimetry for differentiation of these states is related both with physical and applied reasons. As to physical reasons, interest stems from the possibility to ascertain mechanisms of transformation of the optical-andgeometrical structure observed in polycrystalline network inherent to epithelial and connective tissues of rectum wall. Benign changes are accompanied by formation of definite spatially oriented directions for protein fibrils. In other words, there formed are definite statistically probable parts in distributions describing orientations of optical axes observed in biological crystals. In the case of malignant changes, the above asymmetry in orientation structure is increased with simultaneous enhancement in optical anisotropy due to the increased concentration of proteins in newly formed fibrilar structures-processes of a tumor.

Fig. 6 illustrates capabilities of the crosscorrelation analysis aimed at distributions of polarization azimuths in the field of laser radiation transformed by histological sections of tissue biopsy, when differentiating benign and malignant changes.

In the case of malignant tumor processes, there formed is a sub-ensemble of birefringent fibrils with spatially determinate directions of optical axes. As a consequence, coordinate-homogeneous distributions of the polarization azimuth are formed. In the case of malignant tumor, coordinate homogeneity of polarization azimuth values is lower. Respectively, autocorrelation dependences obtained for the direction close to the growth one possess higher velocity of decay and lower halfwidth.

Quantitatively, the above differences are characterized by average values and standard deviations in the set of cross-correlation moments of the second and fourth orders, which is summarized in Table 2.

Table 2. Cross-correlation moments for distributions of polarization azimuths

\begin{tabular}{|c|c|c|}
\hline $\mathrm{dK}_{\mathrm{i}}$ & Benign tumor & Malignant tumor \\
\hline $\mathrm{dK}_{2}$ & $0.83 \pm 0.08$ & $1.79 \pm 0.13$ \\
\hline $\mathrm{dK}_{4}$ & $0.91 \pm 0.07$ & $2.84 \pm 0.18$ \\
\hline
\end{tabular}

It has been ascertained that the cross-correlation moment of the fourth order is the most sensitive in differentiation of Fourier spectra corresponding to laser images of histological sections taken from benign and malignant changed tissue of rectum. The differences of these values reach three times.

\section{The method of consistent spatial-frequency filtration of polarization-inhomogeneous images of biological layers}

To determine the capabilities of the method for Fourier polarimetry with spatial-frequency filtration applied to optical separation of linear and circular birefringence manifestations, we performed computer modeling by using the network of birefringent polycrystalline cylinders with ordered directions of optical axes. 


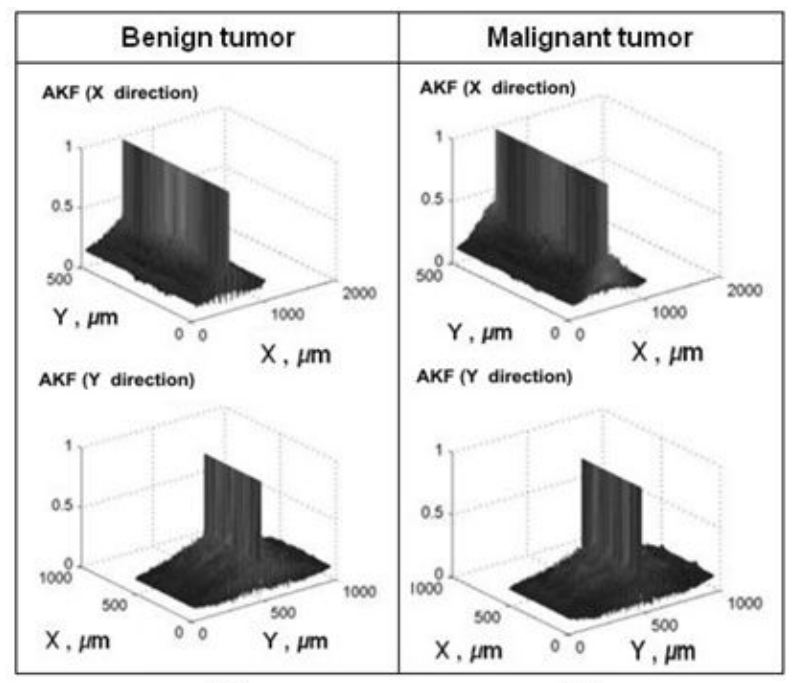

(a)

(b)

Fig. 6. Orthogonal autocorrelation functions of Fourier spectra corresponding to the field of laser radiation scattered by rectum tissue with benign (a) and malignant (b) changes.

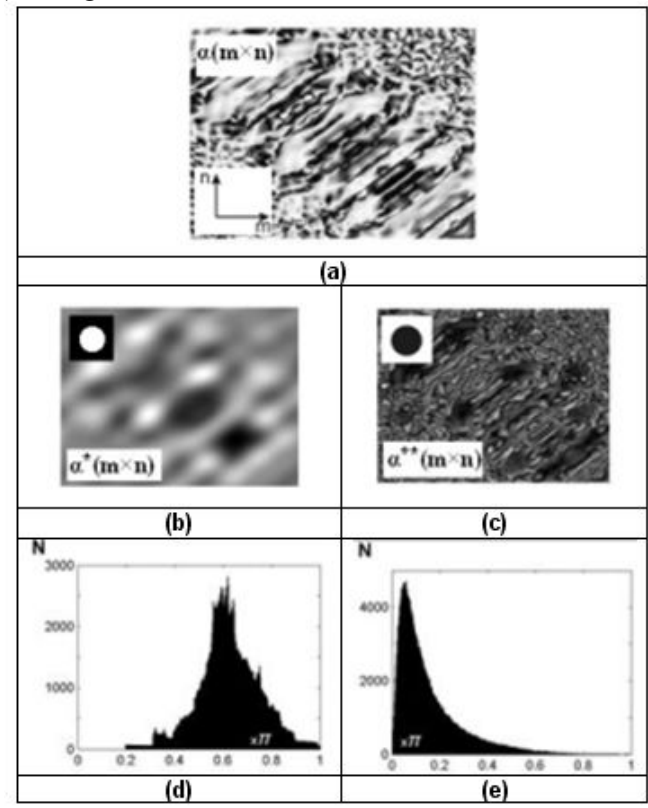

Fig. 7. Spatial-frequency filtration of the polarization map corresponding to the network of birefringent cylinders.

Using computer modeling, we studied the efficiency of consistent spatial-frequency filtration when obtaining polarization maps of polycrystalline networks with linear (network of cylinders) and circular (network of globes) birefringence. In this case, we used a special vignetting diaphragm placed in the Fourier plane. Fig. 7 shows a series of respective coordinate $(a, b, c)$ and probabilistic (d, e) distributions $\alpha(m \times n)$.

The obtained data illustrate the possibility to efficiently differentiate the low-frequency (a, c) and high-frequency $(b, d)$ components of distributions for polarization azimuths formed in accord with different birefringence mechanisms.

Depicted in Figs 8 and 9 is the series of probabilistic (b, f), correlation (c, g) and spectral (d, h) dependences that characterize the coordinate distributions for polarization azimuths (a, e) of largescale (Fig. 8) as well as small-scale (Fig. 9) networks of proteins in the films of blood plasma taken from a healthy man $(\mathrm{a}-\mathrm{d})$ and that sick $(\mathrm{e}-\mathrm{h})$ with prostate cancer.

It has been ascertained that histograms for the distribution of polarization azimuths in blood plasma film images for the healthy man possess more asymmetric structure (Fig. $8 \mathrm{~b}$ ) as compared to the same distribution for the patient sick with stomach cancer (Fig. 9f). The revealed feature is related with a higher order among directions of optical axes in the structure of networks of needle-like albumin crystals in the blood plasma taken from the group 1 than that from the group 2. This geometric structure is inherent to formation of priority, the most probable among all the possible values 


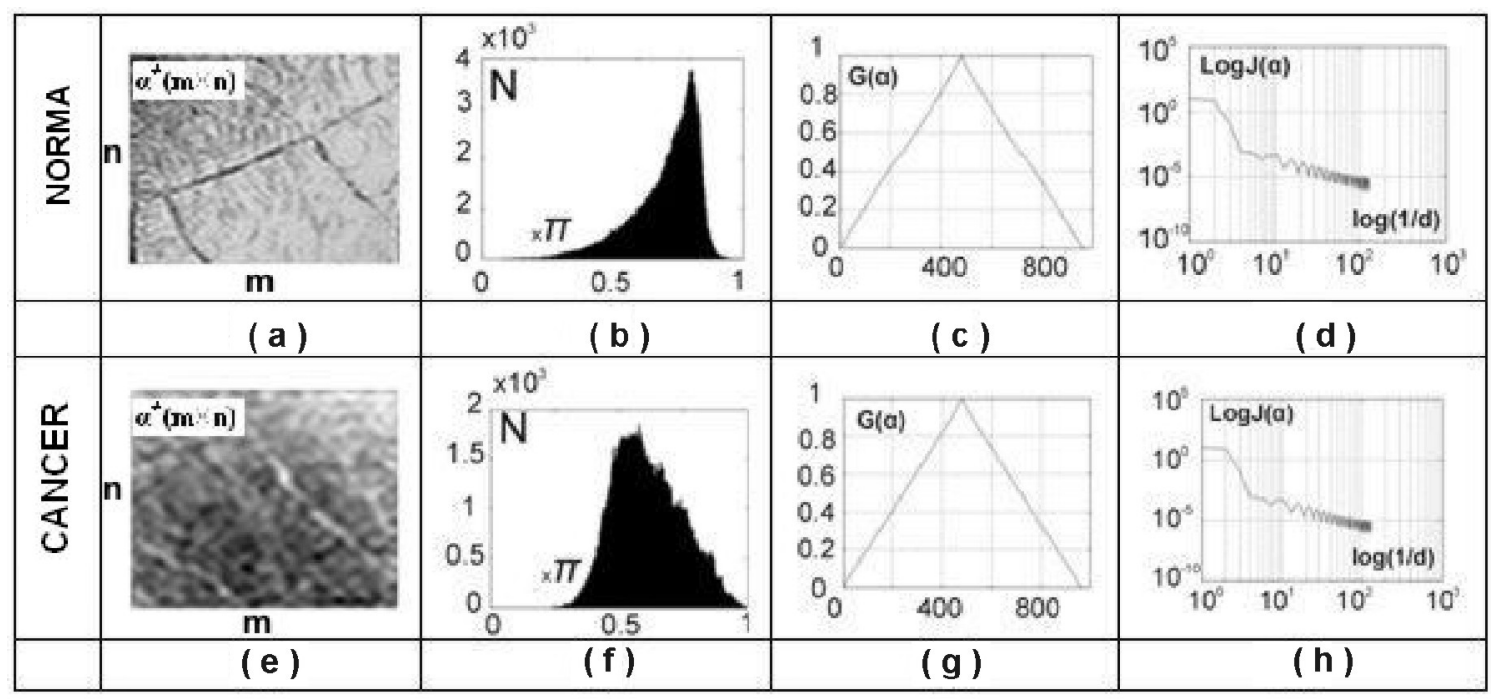

Fig. 8. Large-scale polarization maps of the linearly birefringent component of proteins in blood plasma films and their statistical, correlation and fractal parameters. See explanations in the text.

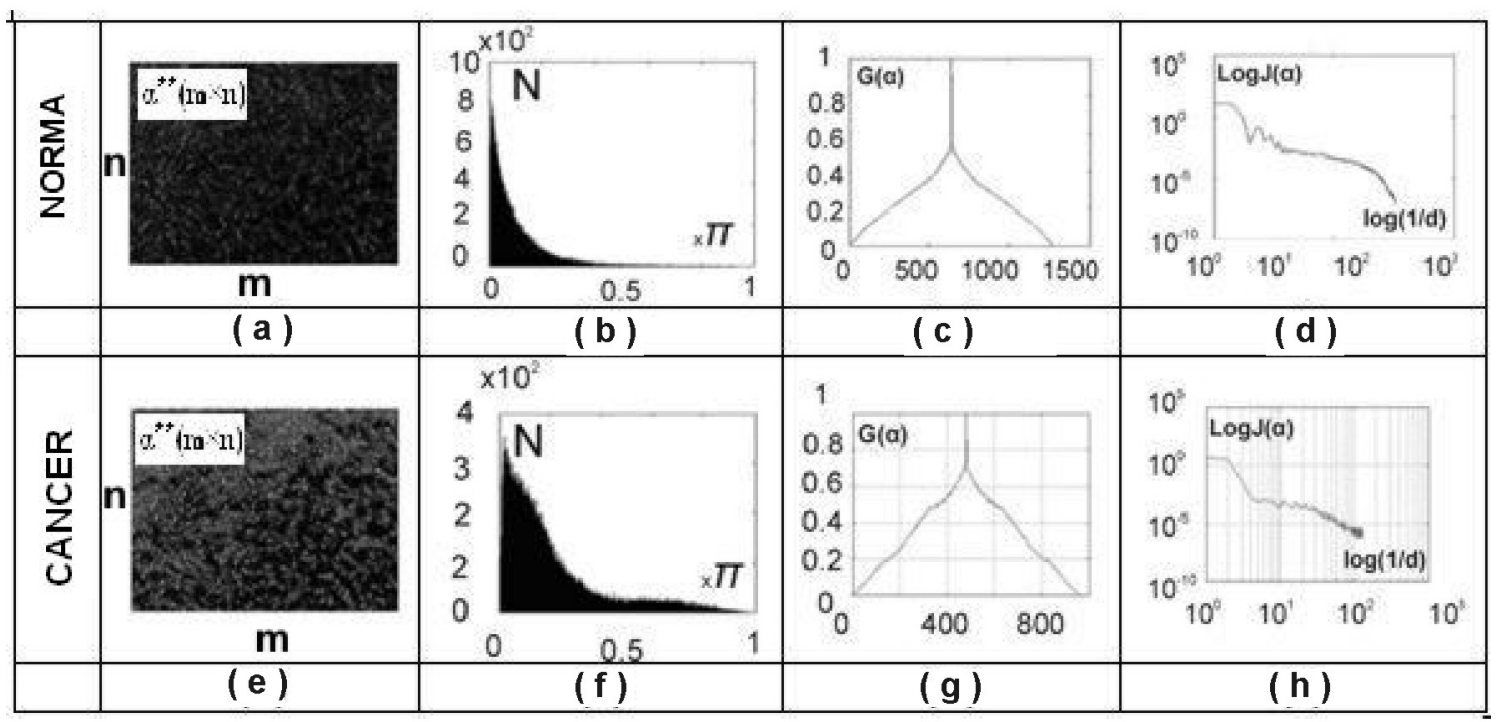

Fig. 9. Small-scale polarization maps for the circular birefringent component of proteins in blood plasma films and their statistical, correlation and fractal parameters. See explanations in the text.

of polarization azimuths, the set of which forms the main extreme.

Dependences of autocorrelation functions for polarization azimuth distributions of both samples of blood plasma films decay smoothly and monotonically (Figs $8 \mathrm{c}$ and $8 \mathrm{~g}$ ). This regularity is indicative of the coordinate homogeneous structure for albumin crystal polarization maps. Besides, we revealed a scale-selfsimilar structure of these maps - logarithmic dependences of the power spectra for polarization azimuth distributions are characterized by the same slope angle practically within the whole range from 2 up to $1000 \mu \mathrm{m}$ (Figs $8 \mathrm{~d}$ and $8 \mathrm{~h}$ ).

It has been ascertained that the main feature of oncological changes is formation of definite spatially determinate directions of orientation of albumin needlelike crystals and, related with it, transformation of the histogram for the distribution of polarization azimuth values.

Another picture takes place when using the complex statistical, correlation and fractal analysis of coordinate distributions for polarization azimuths corresponding to optically active small-scale spherolytic albumin networks.

We revealed the following signs of optical activity transformations related with presence of oncological state. Essential extending the range of changes in random values $\alpha^{* *}$ of the histogram corresponding to the spherolytic polycrystalline network owing to growth of the globulin concentration (Figs $9 \mathrm{~b}$ and 9f).

\section{(C) 2012, V. Lashkaryov Institute of Semiconductor Physics, National Academy of Sciences of Ukraine}


Table 3. Statistical, correlation and fractal parameters for different scale components of polarization maps corresponding to blood plasma films

\begin{tabular}{|c|c|c|c|c|}
\hline \multirow{2}{*}{ Parametr } & \multicolumn{2}{|c|}{ Albumins $\alpha^{*}(\mathrm{~m} \times \mathrm{n})$} & \multicolumn{2}{c|}{ Globulins $\alpha^{* *}(\mathrm{~m} \times \mathrm{n})$} \\
\cline { 2 - 5 } & Norma & Cancer & Norma & Cancer \\
\hline $\mathrm{M}_{1}$ & $1.07 \pm 0.17$ & $1.12 \pm 0.19$ & $0.27 \pm 0.037$ & $0.35 \pm 0.046$ \\
\hline $\mathrm{M}_{2}$ & $0.14 \pm 0.021$ & $0.19 \pm 0.025$ & $0.082 \pm 0.012$ & $0.19 \pm 0.031$ \\
\hline $\mathrm{M}_{3}$ & $1.73 \pm 0.24$ & $0.16 \pm 0.027$ & $0.11 \pm 0.017$ & $1.03 \pm 0.15$ \\
\hline $\mathrm{M}_{4}$ & $0.89 \pm 0.011$ & $0.12 \pm 0.017$ & $1.54 \pm 0.22$ & $0.63 \pm 0.077$ \\
\hline $\mathrm{Q}_{2}$ & $0.28 \pm 0.045$ & $0.29 \pm 0.047$ & $0.015 \pm 0.0028$ & $0.088 \pm 0.011$ \\
\hline $\mathrm{Q}_{4}$ & $0.045 \pm 0.0077$ & $0.05 \pm 0.009$ & $4.38 \pm 0.74$ & $1.82 \pm 0.31$ \\
\hline $\mathrm{V}(\eta)$ & Fractal & Fractal & Fractal & Multifractal \\
\hline $\mathrm{D}$ & $0.31 \pm 0.055$ & $0.29 \pm 0.051$ & $0.095 \pm 0.013$ & $0.17 \pm 0.028$ \\
\hline
\end{tabular}

"Pathological" growth of the amount of optically active crystals results in formation of a more coordinate homogeneous distribution for $\alpha^{* *}$ values. In the correlation analysis, this regularity is pronounced as a slow drop and wider halfwidths of autocorrelation functions (Figs 9c and 9g). Reconstruction of the globulin polycrystalline network is accompanied by transformation of the practically fractal distribution $\alpha^{* *}$ into the multifractal one - the logarithmic dependence of the power spectrum is characterized by several different slopes of the approximating curve (Fig. 9h).

Quantitatively, these differences between polarization maps are illustrated with the data summarized in Table 3.

Our comparative analysis enabled us to reveal the following parameters diagnostically sensitive to the oncological state of a man's prostate:

- statistical moments of the third and fourth orders for $\hat{\alpha}(m \times n)$ distributions, differences between values of which reach $7\left(Z_{3}\right)$ up to $10\left(Z_{4}\right)$ times;

- statistical moments of the second to fourth orders for $\dot{\alpha}(m \times n)$ distributions, differences between values of which reach $2\left(Z_{2}, Z_{3}\right)$ up to $10\left(Z_{4}\right)$ times;

- statistical moments of the second to fourth orders for autocorrelation functions corresponding to $\dot{\alpha}(m \times n)$ distributions, differences between values of which reach $2.2\left(Q_{2}\right)$ up to $4\left(Q_{4}\right)$ times;

- dispersion $D$ of logarithmic dependences for the power spectra of $\dot{\alpha}(m \times n)$ distributions, differences between values of which reach 2 times;

- transformation of fractal $\dot{\alpha}(m \times n)$ distributions into the multifractal ones.

\section{Conclusions}

1. Offered in this work is the model of generalized anisotropy inherent to protein networks of biological tissues with different scales (polypeptide chains, fibrils, fibers, bunches) and using the computer modeling analyzed have been the main scenarios providing formation and transformation of polarization maps for azimuths and ellipticities of laser images and their Fourier spectra. Also ascertained is their dependence on optical-andgeometrical structure observed in linearly and circularly partial birefringent networks.

2. Developed is the method of Fourier-Stokes polarinetry for spatial-frequency spectra of laser images, which is based on cross-correlation analysis of polarization maps. Using this method, for the first time, we realized differentiation of pathological states observed in biological tissues histological sections prepared from biopsy of benign and malignant tumors of rectum.

3. We have investigated the efficiency of spatialfrequency filtration applied to polarizationinhomogeneous laser images of blood plasma films. For the first time, we realized experimentally the system of consistent Fourier-Stokes polarimetric separation of mechanisms inherent to linear and circular birefringence in polycrystalline albumin and globulin networks of different scales.

4. In approximation of single-step scattering, we found the interrelation between changes of parameters describing anisotropy of linearly (direction of the optical axes and phase shift between orthogonal linearly polarized unit vectors) and circularly (phase shift between circularly polarized unit vectors) polarized amino acid crystals in blood plasma as well as variations of values characteristic for statistical, correlation and spectral moments of the first to fourth orders that characterize coordinate distributions for polarization azimuths and ellipticities, which are formed by partial networks of albumins and globulins in human blood plasma.

\section{References}

1. Tuchin V. V. Handbook of coherent-domain optical methods. Biomedical diagnostics, environmental and material science / edited by Tuchin V. V. Boston. : Kluwer Academic Publishers, 2004. - P. 868. 
2. Handbook of Optical Coherence Tomography / edited by B. E. Bouma and G. J. Tearn // Polarization-sensitive optical coherence tomography / J. F. de Boer, T. E. Milner, M. G. Ducros, S. M. Srinivas and J. S. Nelson. - New York : Marcel Dekker Inc., 2002. - P. 237-274.

3. Sankaran. V. Comparison of polarized-light propagation in biological tissue and phantoms / V. Sankaran, M. J. Everett, D. J. Maitland, J. T. Walsh // Opt. Lett. - 1999. - Vol. 24. - P. 1044-1046.

4. Pierce M.C. Birefringence measurements in human skin using polarization-sensitive optical coherence tomography / M.C. Pierce, J. Strasswimmer, B. Hyle Park, B. Cense, J. F. de Boer // J. Biomed. Opt. - 2004. - Vol. 9. - P. 287-291.

5. Angelsky O. V. Investigation of 2D Mueller matrix structure of biological tissues for preclinical diagnostics of their pathological states / O. V. Angelsky, Yu. Ya. Tomka, A. G. Ushenko, Ye. G. Ushenko, Yu. A. Ushenko // J. Phys. D: Appl. Phys. - 2005. - Vol. 38. - P. 4227-4235.

6. Ushenko A. G. Evolution of statistic moments of 2D- distributions o $\mathrm{f}$ biological liquid crystal netmueller matrix elements in the process of their birefringent structure changes. / A. G. Ushenko, I. Z. Misevich, V. Istratiy, I. Bachyns'ka, A. P. Peresunko, O. K. Numan, T. G. Moiysuk // Advances in Optical Technologies. - 2010. - ID 423145.

7. O.V. Angelsky. Polarization singularities of the object field of skin surface / O.V. Angelsky, A. G. Ushenko, Yu. A. Ushenko, Ye. G. Ushenko // J. Phys. D: Appl. Phys. - 2006. - Vol. 39. - P. 3547 3558 .

8. Yermolenko S. Spectropolarimetry of cancer change of biotissues / Yermolenko S., Ushenko A., Ivashko P., Goudail F., Gruia I., Gavrilă C., Zimnyakov D., Mikhailova A. // Proceedings of SPIE. - 2009. .- Vol. 7388. - P 73881D.

9. Ushenko A. Statistical and fractal approaches in laser polarimetry diagnostics of the cancer prostate tissues / Ushenko A., Yermolenko S., Prydij A., Guminetsky S., Gruia I., Toma O., Vladychenko K // Proceedings of SPIE. - 2008. .- Vol. 7008. - P 70082C.

10. Laser Speckle and Related Phenomena / edited by J. C. Dainty // Statistical properties of laser speckle patterns / J.W. Goodman. - Berlin : SpringerVerlag, 1975. - P. 9-75. 